>Le trouble déficit de l'attention/hyperactivité (TDAH) est une affection fréquente, associée à des difficultés de fonctionnement psychosocial durables et à une morbidité psychiatrique élevée. Le TDAH est un syndrome hétérogène dans sa présentation clinique et probablement dans son étiologie. II peut être compris comme un spectre de dysfonctionnements cognitifs, émotionnels et comportementaux d'origine multifactorielle, sous l'influence de facteurs génétiques et environnementaux, principalement périnataux. Depuis plusieurs décennies, l'hypothèse d'un dysfonctionnement dopaminergique est l'une des pistes possibles dans la physiopathologie du TDAH, mais d'autres systèmes de neurotransmission semblent aussi jouer un rôle, comme en témoigne le développement thérapeutique récent des agonistes noradrénergiques dans cette indication. Les symptômes cardinaux du TDAH peuvent être améliorés par des interventions psycho-comportementales et des traitements médicamenteux dont les plus utilisés sont les psychostimulants (méthylphénidate et dérivés amphétaminiques). Cependant, le développement des travaux de recherche clinique et neuro-scientifique concernant le TDAH dépasse de loin le seul cadre de la psychopharmacologie. Cet article propose une synthèse des recherches dans les domaines de la génétique et de l'imagerie, approches qui ont permis des avancées prometteuses dans la compréhension des mécanismes de ce syndrome complexe. <

\section{Neurobiologie du trouble déficit de l'attention/ hyperactivité}

Diane Purper-Ouakil, Aude-Marie Lepagnol-Bestel, Edith Grosbellet, Philip Gorwood, Michel Simonneau

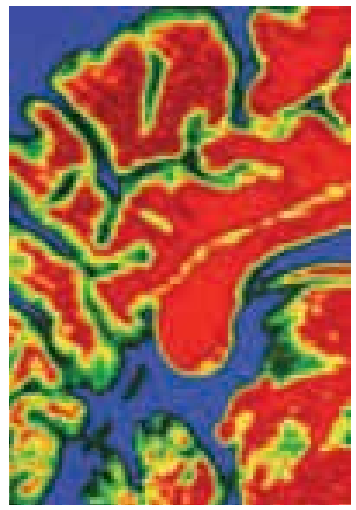

D. Purper-Ouakil : Inserm U894, Centre de psychiatrie et neurosciences, Équipe 1, Analyse génétique et clinique des comportements addictifs et psychiatriques,

2 ter, rue d'Alesia, 75014 Paris, France ; AP/HP, hôpital Robert Debré, service de psychopathologie de l'enfant et de l'adolescent, Paris, France.

diane.purper-ouakil@rdb.aphp.fr A.M. Lepagnol-Bestel : Inserm U894, Centre de psychiatrie

il se manifeste par une impulsivité, des problèmes dans l'anticipation et l'organisation des tâches, des difficultés de maintien de l'attention, et une instabilité motrice de degré variable. La prévalence calculée à partir d'une revue systématique de la littérature incluant 102 études épidémiologiques provenant de tous les continents est de 5,29\% [1]. Il s'agit d'un trouble chronique, associé à un retentissement scolaire, social et familial chez l'enfant, et dont la gêne fonctionnelle est et neurosciences, Équipe 1, Analyse génétique et clinique des comportements addictifs et psychiatriques,

2 ter, rue d'Alesia 75014 Paris, France.

M. Simonneau : Inserm U894, Centre de psychiatrie et neurosciences, 2 ter, rue d'Alésia, 75014 Paris, France. michel.simonneau@inserm.fr P. Gorwood : Inserm U894, Centre de psychiatrie et neurosciences, Équipe l, Analyse génétique et clinique des comportements addictifs et psychiatriques;

CMME, hôpital Sainte-Anne, Université Paris Descartes, Paris, France.

غ́. Grosbellet : École normale supérieure de Cachan,

Cachan, France.

\section{Le trouble déficit de l'attention/hyperactivité (TDAH) : définition, aspects cliniques}

Le TDAH se caractérise par la combinaison de troubles de l'attention et de difficultés de contrôle sur le plan cognitif, émotionnel et comportemental. Cliniquement, encore repérable chez plus de $60 \%$ des adultes concernés [2]. Le TDAH est souvent associé à une comorbidité psychiatrique (troubles oppositionnels et des conduites, troubles anxieux), à des difficultés d'apprentissage et constitue un facteur de risque pour l'abus et la dépendance aux substances psychoactives [3]. Le 
retentissement sur l'entourage familial et les trajectoires scolaires et professionnelles ainsi que les coûts sanitaires et sociaux sont des conséquences indirectes du TDAH [4, 5].

La classification américaine DSM-IV-TR définit le «trouble déficit de l'attention/hyperactivité » comme une entité diagnostique multi-

${ }^{1}$ Diagnostic and statistical manual, fourth edition, text revision ; le DSM est la classification des maladies mentales la plus importante et la plus utilisée. Elle est réalisé par l'American psychiatric association. Le DSM-IV a été publié en 1994, et le texte est corrigé lorsque de nouvelles données ont fait l'objet de publications. dimensionnelle, regroupant trois formes cliniques: le TDAH mixte (inattention et impulsivité/hyperactivité), le TDAH avec inattention prédominante et le TDAH avec hyperactivité/impulsivité prédominante. L'ICD-10² propose une définition plus restreinte du syndrome, celle

\section{A. Présence soit de 1 , soit de 2 :}

1. Six (ou plus) des symptômes suivants d'inattention ont persisté pendant au moins six mois, à un degré qui est inadapté et ne correspond pas au niveau de développement de l'enfant :

Inattention:

- souvent, ne parvient pas à prêter attention aux détails, ou fait des fautes d'étourderie dans les devoirs scolaires, le travail ou d'autres activités ;

- a souvent du mal à soutenir son attention au travail ou dans les jeux ;

- semble souvent ne pas écouter quand on lui parle personnellement ;

- souvent, ne se conforme pas aux consignes et ne parvient pas à mener à terme ses devoirs scolaires, ses tâches domestiques ou ses obligations professionnelles (cela n'est pas dû à un comportement d'opposition, ni à une incapacité à comprendre les consignes) ;

- a souvent du mal à organiser ses travaux ou ses activités ;

- souvent, évite, a en aversion, ou fait à contrecœur les tâches qui nécessitent un effort mental soutenu (comme le travail scolaire ou les devoirs à la maison);

- perd souvent les objets nécessaires à son travail ou à ses activités (par exemple, jouets, cahiers de devoirs, crayons, livres ou outils) ;

- souvent se laisse facilement distraire par des stimulus externes ;

- a des oublis fréquents dans la vie quotidienne.

2. Six (ou plus) des symptômes suivants d'hyperactivité-impulsivité ont persisté pendant au moins six mois, à un degré qui est inadapté et ne correspond pas au niveau de développement de l'enfant :

Hyperactivité:

- remue souvent les mains ou les pieds, ou se tortille sur son siège ;

- se lève souvent en classe ou dans d'autres situations où il est supposé rester assis ;

- souvent, court ou grimpe partout, dans des situations où cela est inapproprié (chez les adolescents et les adultes, ce symptôme peut se limiter à un sentiment subjectif d'impatience motrice) ;

- a souvent du mal à se tenir tranquille dans les jeux ou dans les activités de loisir ;

- est souvent «sur la brèche » ou agit souvent comme s'il était « monté sur ressorts »;

- parle souvent trop.

Impulsivité :

- laisse souvent échapper la réponse à une question qui n'est pas encore entièrement posée ;

- a souvent du mal à attendre son tour ;

- interrompt souvent les autres ou impose sa présence (par exemple, fait irruption dans les conversations ou dans les jeux).

B. Certains des symptômes d'hyperactivité-impulsivité ou d'inattention ayant provoqué une gêne fonctionnelle étaient présents avant l'âge de sept ans.

C. Présence d'un certain degré de gêne fonctionnelle liée aux symptômes dans deux ou plus de deux types d'environnement différents (par exemple, à l'école, au travail, à la maison).

D. On doit mettre clairement en évidence une altération cliniquement significative du fonctionnement scolaire, social ou professionnel.

$\varepsilon$. Les symptômes ne surviennent pas exclusivement au cours d'un trouble envahissant du développement, d'une schizophrénie ou d'un autre trouble psychotique, et ils ne sont pas mieux expliqués par un autre trouble mental (par exemple, trouble thymique, trouble anxieux, trouble dissociatif ou trouble de la personnalité).

Tableau I. Classification des maladies mentales DSM-IV-TR - Critères diagnostiques du trouble : déficit de l'attention-hyperactivité. 


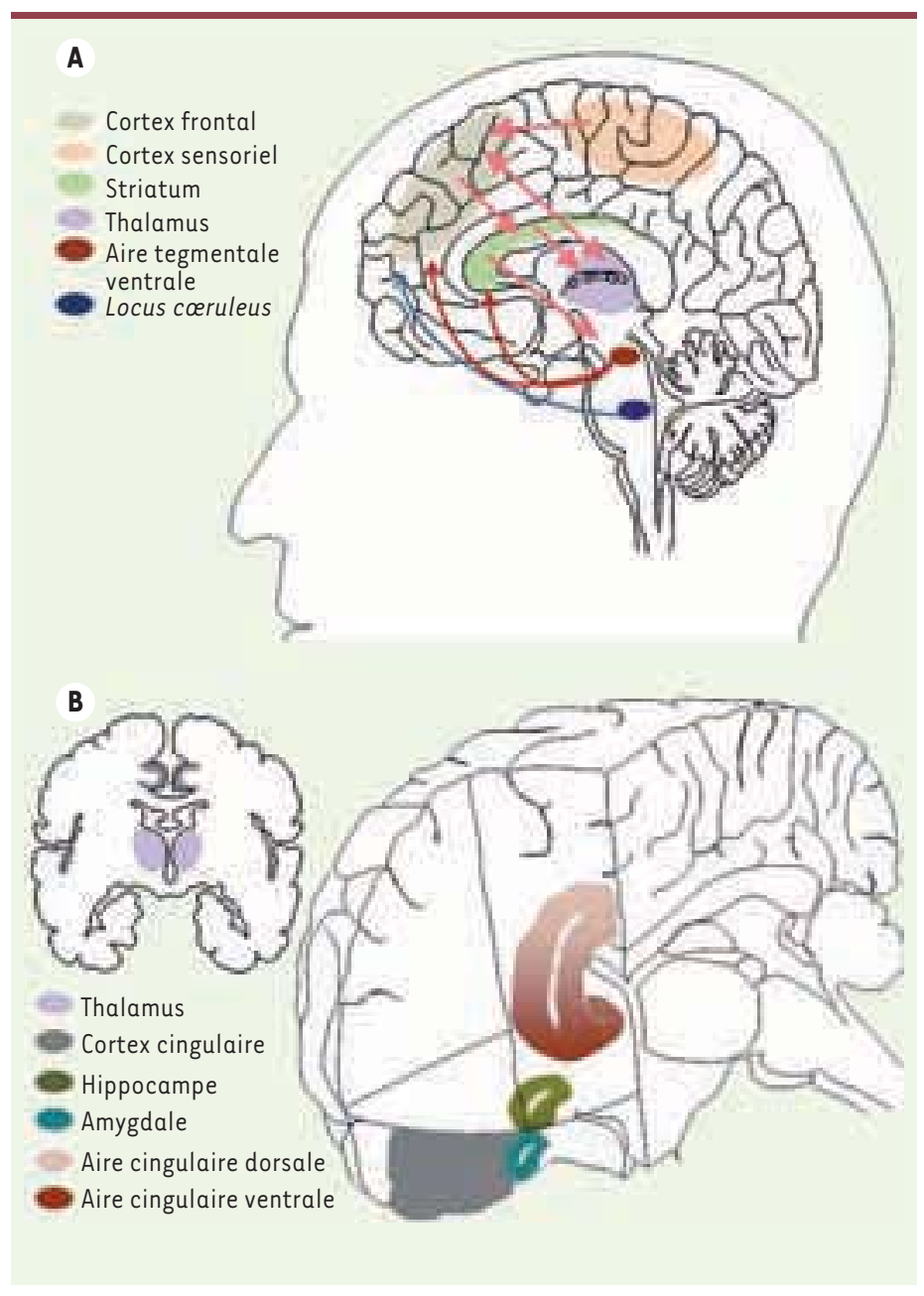

du «trouble hyperkinétique » [F 90] qui exige la présence concomitante de symptômes d'inattention, d'impulsivité et d'hyperactivité (Tableau I).

\section{Hypothèses physiopathologiques}

\section{Les facteurs de risque potentiels}

La surreprésentation du TDAH dans les familles de sujets atteints est bien étayée. Les études de jumeaux ont permis d'estimer que l'héritabilité du TDAH est de $76 \%$, le reste de la variance étant essentiellement attribuable à l'environnement non partagé [6]. L'héritabilité comprend l'ensemble des facteurs génétiques additifs, c'est-à-dire le poids des différents gènes, de leur interaction mutuelle et de leur interaction avec l'environnement. Cette estimation ne donne aucune indication sur les causes du TDAH. Les dimensions telles que l'impulsivité, l'inattention et l'activité motrice sont influencées par des facteurs génétiques communs à la population normale et aux sujets ayant un TDAH. Les facteurs environnementaux incriminés dans la vulnérabilité au TDAH concernent en premier lieu la période périnatale. Les études cas-contrôles montrent que l'exposition prénatale au tabac est associée à un risque deux fois plus important de TDAH chez les enfants
Figure 1. Représentation schématique des régions cérébrales impliquées dans le TDAH. A. Illustration des connexions cortico-striatales, sensori-frontales et des interactions thalamocingulaires. De plus, les structures corticales sont modulées par des afférences noradrénergiques et dopaminergiques provenant du tronc cérébral (respectivement locus coeruleus et aire tegmentale ventrale). B. Illustration de la localisation du cortex cingulaire avec les aires corticales cingulaires dorsales et ventrales qui interagissent avec d'autres structures du système limbique, en particulier l'amygdale et l'hippocampe.

exposés, sans que les différents facteurs confondants (niveau socioéconomique, QI, TDAH parental, abus d'alcool, poids de naissance, etc.) n'expliquent complètement ce résultat [7]. Une étude de jumeaux a montré que si la majeure partie de la variance du TDAH est en rapport avec des facteurs génétiques communs, l'association avec l'exposition au tabac reste toutefois significative [8]. Parmi les autres facteurs incriminés figurent l'exposition prénatale à l'alcool et à d'autres substances psychoactives, la prématurité et le faible poids de naissance, le stress et les carences alimentaires maternelles pendant la grossesse, l'exposition aux polychlorobiphényls ( $P C B)$, à l'hexachlorobenzène et au plomb [9]. Les lésions cérébrales acquises (traumatiques ou post-encéphalitiques) peuvent également s'accompagner de symptômes de TDAH. Des facteurs psychosociaux ont également été associés au TDAH (attitudes parentales hostiles, dépression maternelle), mais la direction de la causalité n'est pas établie du fait de multiples facteurs confondants (par exemple l'existence d'un TDAH chez le parent peut influencer son style éducatif).

\section{Les endophénotypes neuropsychologiques}

Le TDAH étant un trouble hétérogène du point de vue clinique et probablement étiologique, la recherche d'endophénotypes (voir glossaire) cliniques et cognitifs pourrait faciliter les rapprochements entre l'expression des symptômes et leurs mécanismes biologiques sous-jacents. Dans le TDAH, cette approche a surtout été utilisée avec des caractéristiques neuropsychologiques, du fait des déficits exécutifs et d'inhibition motrice associés à ce trouble. Selon un modèle récent, le TDAH serait le produit de trajectoires neurodéveloppementales différentes. L'une serait sous-tendue par un dysfonctionnement du système de régulation top-down fronto-exécutif ${ }^{3}$, se

\footnotetext{
${ }^{3}$ || existe deux systèmes d'attention. L'un, top down, est endogène (donc volontaire) et dirigé vers un but. II implique le système dorso-fronto-pariétal. Le second, bottom up, exogène (automatique et involontaire), part des stimulus (informations
} sensorielles) vers les aires supérieures du cerveau.

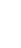




\section{GLOSSAIRE}

Empreinte génomique : un gène est soumis à empreinte lorsque l'expression de ce gène dépend de son origine parentale (maternelle ou paternelle).

Endophénotype : un endophénotype (ou phénotype intermédiaire) est un marqueur, clinique, cognitif ou biologique, de la vulnérabilité à pendant de l'expression clinique de la vulnérabilité et existe donc également chez les apparentés non atteints.

Héritabilité : I'héritabilité correspond au «pourcentage d'explication » de la maladie dû aux différences interindividuelles du génome. Elle comprend les facteurs génétiques additifs, c'est-à-dire le poids des différents gènes, celui de leur interaction mutuelle ainsi que les interactions entre gènes et environnement. Trois composantes principales sont étudiées dans les modèles les plus simples: les facteurs génétiques $\left(h^{2}\right)$, l'environnement partagé $\left(c^{2}\right)$ (commun à tous les membres d'une fratrie) et l'environnement non partagé $\left(e^{2}\right)$ (unique à chaque individu de la famille). La concordance pour un trait donné entre jumeaux monozygotes correspond à la somme des effets génétiques et d'environnement partagé, puisque l'on considère dans ce modèle que les différences entre jumeaux monozygotes élevés ensemble sont dues à l'environnement non partagé. Le calcul de l'héritabilité ne permet cependant qu'une estimation relativement approximative; tout au plus ce critère permet-il de juger de la proportion de facteurs génétiques additifs influençant un trait phénotype. II n'est pas exempt de biais : il n'est pas exclu par exemple que les jumeaux monozygotes aient un environnement plus similaire que les jumeaux dizygotes, ce qui pourrait conduire à une surestimation des facteurs génétiques. Les modèles les plus récents et les approches complémentaires (études d'adoption) permettent de contrôler une partie des facteurs de confusion.

Méta-analyse: une méta-analyse est une démarche statistique combinant les résultats d'une série d'études indépendantes sur un problème donné. La méta-analyse permet une analyse plus précise des données par l'augmentation du nombre de cas étudiés. Elle permet aussi de détecter les biais de méthode des études analysées. L'OR (odds ratio) se définit comme le rapport des risques qu'un événement présent dans un groupe de personnes $A$ s'observe également dans un groupe B. S'il ne se distingue pas statistiquement de l (c'est-à-dire que son intervalle de confiance englobe 1 ), la maladie est indépendante du groupe. S'il est supérieur à l, la maladie est plus fréquente dans le groupe $A$. un trouble et qui possède une héritabilité supérieure à 0 . II est indé-

\section{Les mécanismes neurobiologiques}

Le rôle des catécholamines dans les syndromes hyperkinétiques de l'enfant a été mis en avant dès les années 1970, après la découverte de l'effet thérapeutique des psychostimulants, principaux traitements médicamenteux du trouble déficitaire de l'attention/hyperactivité. Ainsi, le méthylphénidate (Ritaline ${ }^{\circledast}$, Concerta $^{\circledast}$ ) augmente la concentration de dopamine dans la fente synaptique par l'intermédiaire du blocage du transporteur de la dopamine (DAT). Les données d'imagerie fonctionnelle montrent que cette augmentation de dopamine extracellulaire est plus grande après exposition à un stimulus environnemental ayant une valeur de renforcement (test mathématique) qu'après un stimulus neutre [12]. L'hypothèse proposée par les auteurs serait que l'augmentation extracellulaire de dopamine au niveau des neurones striataux diminuerait leur activité de base aléatoire et permettrait aux afférences cortico-striatales liées aux stimulus pertinents d'être plus efficaces (Figure 1).

Les études neuropharmacologiques successives et la découverte de l'action thérapeutique des agonistes noradrénergiques ont conduit à affiner l'hypothèse de déplétion dopaminergique incriminée dans le défaut d'inhibition et dans les anomalies du système de renforcement décrits dans le TDAH [13]. La noradrénaline, via les récepteurs postsynaptiques alpha2A localisés sur les épines dendritiques des neurones glutamatergiques, augmente les réponses synaptiques du réseau préfrontal. Les molécules noradrénergiques telles que l'atomoxétine et la guanfacine, en dehors d'une amélioration des capacités attentionnelles et de mémoire de travail via leur action sur le cortex préfrontal, faciliteraient l'action inhibitrice de ce dernier sur le cortex moteur et les régions sous-corticales [14]. Comme l'ont montré les études d'imagerie du striatum, on s'attend à ce que la dopamine augmente le rapport signal pertinent/bruit de fond au niveau des neurones glutamatergiques du cortex préfrontal via l'activation des récepteurs Dl [15]. L'ensemble de ces données suggère une base neurobiologique complexe, dépassant

traduisant par un déficit des capacités d'inhibition, l'autre mettrait en jeu des processus motivationnels et émotionnels appartenant à la régulation bottomup illustrés par l'intolérance au délai de récompense (delay aversion) dans les épreuves neuropsychologiques [10]. Une étude de screening du génome a récemment montré deux liaisons significatives pour des traits cognitifs; le premier lie une tâche de temps de réaction avec un locus en $2 q 21.1$, le second une épreuve de mémoire de travail verbale et un locus situé en $13 q 12.11$ [11]. une hypothèse dopaminergique exclusive (pour une revue critique de l'hypothèse dopaminergique voir [16]) et impliquant une diversité de cibles moléculaires (Figures $2 A$ et $2 B$ ).

\section{Les modèles animaux}

Les modèles animaux illustrent la participation de multiples systèmes de neurotransmission dans des phénotypes analogues au TDAH. Van der Kooij et al. [17] rapportent leur analyse de 14 modèles animaux présentant une hyperactivité. Cependant, la description phénotypique des animaux reste parcellaire et la réponse aux psychostimulants est variable. Nous avons choisi 5 modèles animaux génétiques pour 


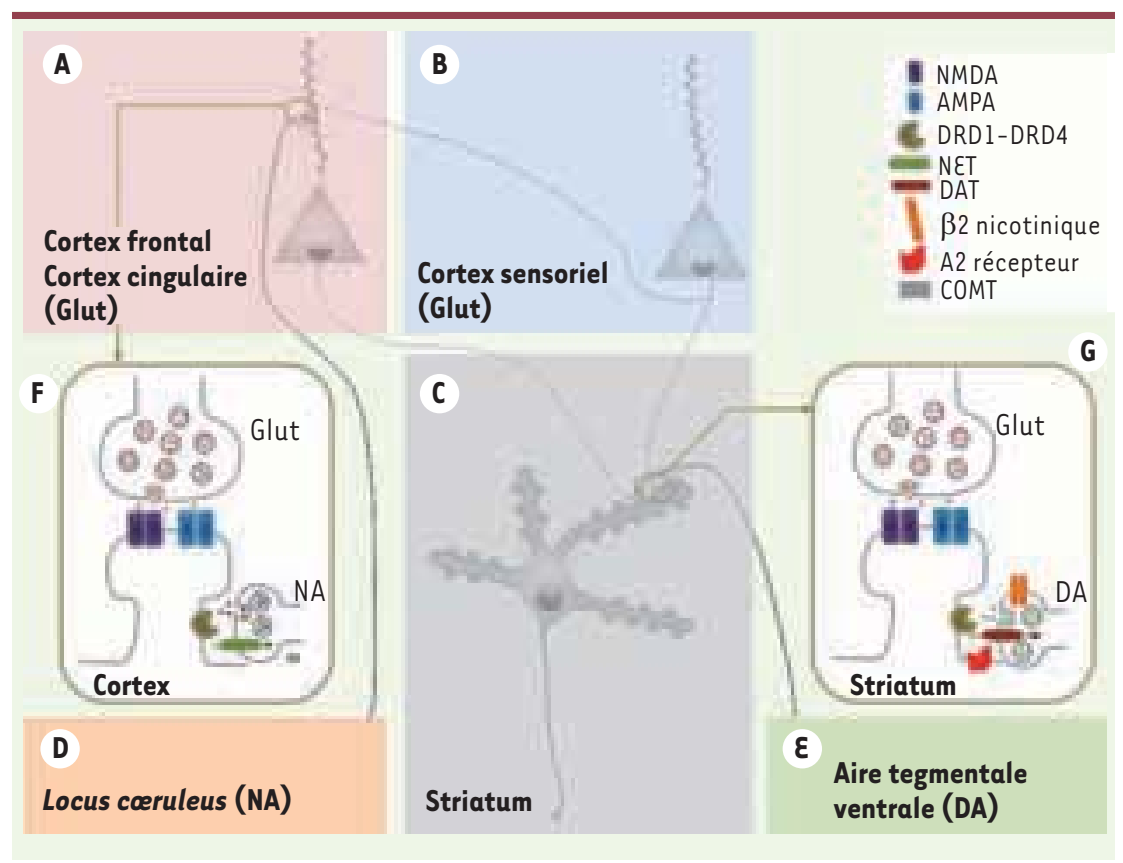

Figure 2. Bases fonctionnelles des synapses clés impliquées dans le TDAH. Représentation de deux neurones corticaux respectivement localisés dans le cortex frontal ou le cortex cingulaire ( $A$ ) et le cortex sensoriel (B) et d'un neurone épineux de taille moyenne du striatum (c) (medium spiny neuron du striatum). Ces neurones épineux du striatum correspondent à plus de $95 \%$ des neurones totaux du striatum. Les neurones frontaux et cingulaires reçoivent des afférences noradrénergiques du locus coeruleus (D). Les neurones striataux reçoivent des afférences de l'aire tegmentale ventrale $(\varepsilon)$. Deux synapses clés sont schématisées: une synapse glutamatergique du cortex frontal ou cingulaire, correspondant aux afférences corticales sensorielles sur ces deux aires, et une synapse glutamatergique du striatum, correspondant aux afférences corticales sur le striatum. Au niveau de la synapse corticale $(F)$, des récepteurs NMDA et AMPA sont localisés dans la membrane de l'épine dendritique. La noradrénaline est libérée au niveau de varicosités localisées à proximité de la tige des épines dendritiques et agit sur des récepteurs métabotropiques (récepteur A2). La NA est recaptée par des transporteurs localisés dans la membrane des varicosités (NET). L'enzyme de dégradation des catécholamines, la catécholamine-0-méthyl-transférase (COMT) est localisée dans les astrocytes. Au niveau de la synapse striatale (G), des récepteurs NMDA et AMPA sont localisés dans la membrane de l'épine dendritique. La dopamine est libérée au niveau de varicosités localiséees à proximité de la tige des épines dendritiques et agit sur des récepteurs métabotropiques (récepteur DI à D4). La dopamine est recaptée par des transporteurs localisés dans la membrane des varicosités (DAT). Ces varicosités libérant la dopamine présentent également des récepteurs-canaux cholinergiques ayant en particulier des sous-unités de type $\beta 2$.

illustrer la diversité des mécanismes neurobiologiques qui ont été proposés comme sous-jacents aux TDAH. Ils impliquent les récepteurs dopaminergiques, noradrénergiques et nicotiniques. Notons que ces récepteurs peuvent être dérégulés d'une façon très indirecte comme c'est le cas pour les souris Snap25 (synaptosomal-associated protein 25) et Fmrl (fragile X mental retardation 1) (Tableau II).

Excepté les modèles basés sur des manipulations génétiques, des modifications de l'environnement peuvent induire une hyperactivité chez l'animal. Par exemple, l'exposition post-natale aux PCB entraîne une hyperactivité chez le rat. Les modifications transcriptionnelles induites par les PCB sont similaires à celles retrouvées chez le rat SHR (spontaneously hypertensive rat) (Tableau II) et concernent des gènes codant des protéines impliquées dans le remodelage de la chromatine [18].

\section{Études de génétique moléculaire}

\section{Études d'association}

Compte tenu des données pharmacologiques, les premiers gènes candidats sélectionnés dans les études d'association sont impliqués dans la neurotransmission catécholaminergique. Les données des études d'association figurent dans le Tableau III. II faut noter que les polymorphismes dont l'association avec le TDAH est répliquée ne rendent compte que d'une faible proportion de la variance du phénotype, ce qui plaide pour un modèle multigénique dans lequel certains gènes pourraient être associés à des formes cliniques particulières. Par exemple, l'influence du gène SLC6A3/DATI (solute carrier family 6) semble plus importante pour les sujets TDAH sans trouble des conduites associé [19]. La récente étude collaborative IMAGE ne montrant pas d'association au-delà du seuil de significativité défini pour les études d'association sur le génome entier $\left(p<5 \times 10^{-8}\right)$, il semble prématuré de conclure de façon définitive à l'implication de certains gènes candidats, même en présence d'une méta-analyse positive [20] (Tableau III).

\section{Études de criblage du génome}

Cette approche examine de nombreux marqueurs de I'ADN afin de déterminer si certaines régions chromosomiques sont plus souvent partagées par des membres atteints dans les familles TDAH. Les sept études de criblage du génome réalisées chez des paires de germains, des familles multiplex ou dans des isolats génétiques, montrent des données convergentes pour certaines régions (notamment $5 p 13,9 q 22$ et $16 q 23$ ), mais aucune 


\begin{tabular}{|c|c|c|c|c|c|}
\hline Modèle & $\begin{array}{l}\text { Base } \\
\text { moléculaire }\end{array}$ & $\begin{array}{l}\text { Hyper- } \\
\text { activité }\end{array}$ & Impulsivité & $\begin{array}{l}\text { Stéréo- } \\
\text { typies }\end{array}$ & $\begin{array}{c}\text { Mécanismes } \\
\text { physiopathologiques }\end{array}$ \\
\hline Rat SHR [36] & $\begin{array}{l}\text { Insertion } 160 \mathrm{pb} \\
\text { dans un 3'-UTR }\end{array}$ & + & + & - & $\begin{array}{l}\text { Synapses dopaminergiques } \\
\text { hypofonctionnelles }\end{array}$ \\
\hline Souris Snap25 $5^{-/-}[37]$ & $\begin{array}{l}\text { K0 du gène Snap25 } \\
\text { (mutant spontané) }\end{array}$ & + & + & - & $\begin{array}{l}\text { Synapses noradrénergiques hyperfonc- } \\
\text { tionnelles }\end{array}$ \\
\hline Souris Dat ${ }^{-1-}[38]$ & KO du gène Dat & + & $?$ & + & $\begin{array}{l}\text { Synapses dopaminergiques hypofonction- } \\
\text { nelles (libération phasique) }\end{array}$ \\
\hline Souris $\beta 2^{-/-}$[39] & $\begin{array}{l}\text { K0 sous-unité } \beta 2 \text { du } \\
\text { récepteur nicotinique }\end{array}$ & + & + & - & $\begin{array}{l}\text { Récepteurs nicotiniques présynaptiques } \\
\text { dont l'ouverture facilite la libération } \\
\text { phasique de dopamine }\end{array}$ \\
\hline Souris Fmrl [40] & KO du gène $F m r l$ & + & + & ? & Dérégulation des récepteurs \\
\hline
\end{tabular}

Tableau II. Modèles animaux. Une hyperactivité peut être obtenue via des modifications du transcrit du gène DATI codant le transporteur de la dopamine (rat SHR) [36], par des modifications de la libération noradrénergique (souris Snap25 $5^{-/}$) [37], par l'invalidation (KO) du gène Dat codant le transporteur de la dopamine [38] des récepteurs nicotiniques $\beta 2$ (souris $\beta 2^{-/-}$) [39] et par dérégulation des récepteurs dopaminergiques (souris $\mathrm{Fmrp}^{-/-}$) [40].

n'a montré de liaison statistiquement significative dans l'ensemble des échantillons. Une méta-analyse de ces études a néanmoins mis en évidence une liaison significative pour une région de 64 à $83 \mathrm{Mb}$ sur le chromosome 16 [21].

Une discordance entre héritabilité élevée et faible proportion de risque attribuable aux gènes identifiés dans les études d'association, notamment celles qui concernent le génome entier, est identifiée dans le TDAH, mais aussi dans d'autres maladies dont l'étiologie est complexe. Une discussion approfondie de la notion d'« héritabilité perdue » et des propositions de nouvelles stratégies d'études génétiques ont été publiées par Manolio et al. [22].

\section{Mécanismes épigénétiques}

Dans toutes les maladies neuropsychiatriques, comme d'une façon plus générale dans les comportements, l'intervention de mécanismes moléculaires liés à l'empreinte parentale génomique (voir glossaire) a été envisagée (voir synthèse de C. Proudhon et D. Bourchis, ce numéro), en rapport avec des modifications chromatiniennes entraînant des modifications d'expression des gènes [23]. Une transmission préférentielle de l'allèle paternel a été mise en évidence pour le gène SNAP25 qui code une protéine impliquée dans la libération du neurotransmetteur [24]. Le modèle murin d'invalidation du gène homologue murin SNAP25 présente des traits phénotypiques rappelant la maladie (Tableau III). Les modifications épigénétiques liées au remodelage de la chromatine qui ont été identifiées dans certaines maladies neuropsychiatriques ainsi que dans l'addic- tion n'ont pas été documentées à ce jour dans le TDAH. Cette voie de recherche est une piste prometteuse, comme le soulignent Mill et Petronis [9].

\section{Études pharmacogénétiques}

Les études des facteurs génétiques impliqués dans les variations individuelles de tolérance et d'efficacité des médicaments se sont principalement centrées sur les polymorphismes des gènes dopaminergiques. Ainsi, le VNTR (variable number of tandem repeat) 3'UTR du gène DATI (SLC6A3) a été le premier polymorphisme impliqué dans l'efficacité du méthylphénidate [25]. Les études ultérieures n'ont pas toutes répliqué ces résultats, probablement du fait de la grande diversité des méthodes employées. Les données issues de notre cohorte clinique d'enfants et d'adolescents ont cependant confirmé l'association du génotype 10 $R / 10-R$ ( $R$ pour répétitions) du VNTR 3'UTR du DATI avec une réponse thérapeutique de moins bonne qualité, selon deux critères de réponse thérapeutique [26]. Une méta-analyse des études pharmacogénétiques ayant étudié ce polymorphisme retrouve également l'association du génotype $10-R / 10-R$ et d'une moindre réponse au traitement avec un $O R$ (odds ratio) de 0,46 (IC95\% 0,28-0,76) [26]. Bien que moins étudiés, d'autres polymorphismes tels que le VNTR DRD4*7 (dopamine $D 4$ receptor), seul [27] ou en interaction avec le variant $L$ de la région promotrice du transporteur de la sérotonine (5-HTTLPR) [28], ont été associés à la réponse au méthylphénidate.

\section{Interactions gène-environnement}

Une difficulté majeure dans l'étude de gènes impliqués dans des troubles comportementaux provient de l'interaction de ces gènes avec l'environnement. Par exemple, le risque de TDAH pour des enfants exposés à la nicotine in utero est plus important si l'enfant a hérité de l'allèle 10-répétitions de DATI [29]. La nicotine, en exposition prénatale, interagirait avec le génotype au niveau d'au moins trois locus 


\begin{tabular}{|c|c|c|c|}
\hline Gène & Allèle à risque le plus étudié & Fonction de la protéine & Confirmé par une méta-analyse \\
\hline \multicolumn{4}{|c|}{ Système dopaminergique } \\
\hline \multirow[t]{2}{*}{ DRD4 } & $\begin{array}{l}\text { - Variant de } 7 \text { répétitions d'un VNTR de } 48 \text { pb } \\
\text { dans l'exon } 3\end{array}$ & Récepteur de la DA & Oui, OR = 1,34 [41] \\
\hline & $\begin{array}{l}\text { - Polymorphisme dans la région promotrice, allèle } \\
521 T>C\end{array}$ & Récepteur de la DA & Oui, OR = 1,21 [42] \\
\hline DRD5 & $\begin{array}{l}\text { Répétition de dinucléotides à environ } 18,5 \text { kb } \\
\text { de l'extrémité } 5 \text { ' }\end{array}$ & Récepteur de la DA & Oui, OR = 1,3 [41] OR = 1,23 [42] \\
\hline \multirow[t]{3}{*}{ SLC6A3/DAT1 } & - Variant de 10 répétitions d'un VNTR en 3' UTR & Transporteur de la DA & Oui, $O R=1,12[42]$ OR = 1,17 [43] \\
\hline & - Variant de 3 répétitions d'un VNTR dans l'intron 8 & Transporteur de la DA & Oui, OR = 1,25 [42] \\
\hline & - Polymorphisme dans la région 3'UTR, allèle 328G>A & Transporteur de la DA & Oui, OR = 1,20 [42] \\
\hline DBH & $\begin{array}{l}\text { Polymorphisme d'un site de restriction par Taql } \\
\text { dans l'intron } 5\end{array}$ & $\begin{array}{l}\text { Enzyme responsable de la conversion de } \\
\text { DA en NA }\end{array}$ & Oui \\
\hline MAO-A & $\begin{array}{l}\text { Variant de } 4 \text { ou } 5 \text { répétitions d'un VNTR de } 30 \text { pb } \\
\text { dans la région promotrice }\end{array}$ & Dégrade NA, DA et 5-HT & Non \\
\hline DRD2/DRD3 & Différents allèles étudiés & Récepteurs de la DA & Non \\
\hline СОMT & $\begin{array}{l}\text { Polymorphisme de Val108Met qui engendre une } \\
\text { forme de l'enzyme de basse/haute activité }\end{array}$ & $\begin{array}{l}\text { Catalyse une étape-clé de la dégrada- } \\
\text { tion de la DA et des catécholamines }\end{array}$ & Non \\
\hline \multicolumn{4}{|c|}{ Système noradrénergique } \\
\hline ADRA2A & SNP dans la région promotrice, allèle $1291 \mathrm{l}>\mathrm{G}$ & Récepteur de la NA & Non \\
\hline ADRA2C & Répétition de dinucléotides à environ 6 kb du gène & Récepteur de la NA & Non \\
\hline SLC6A2/NET & Divers SNP (introns 7, 9, 13 ; exon 9) & Transporteur de la NA & Non \\
\hline \multicolumn{4}{|c|}{ Système sérotoninergique } \\
\hline HTR1B & $\operatorname{SNP}(861 G>C)$ exon 1 & Récepteur de 5-HT & Oui, OR = 1,11 [42] OR = 1,35 [44] \\
\hline HTR2A & Polymorphismes T102C, G1438A, His452Tyr & Récepteur de 5-HT & Non \\
\hline SLC6A4/5HTT & $\begin{array}{l}\text { Polymorphisme d'insertion/délétion de } 44 \text { pb } \\
\text { (HTTLPR) dans la région promotrice }\end{array}$ & Transporteur de $5-\mathrm{HT}$ & $\begin{array}{l}\text { Oui, OR }=1,17[42] \\
\text { OR }=1,31[45]\end{array}$ \\
\hline TPH-2 & Différents SNP & $\begin{array}{l}\text { Enzyme limitante dans la synthèse de } \\
5-\mathrm{HT}\end{array}$ & Non \\
\hline \multicolumn{4}{|c|}{ Autres gènes candidats } \\
\hline SNAP25 & 2 SNP bialléliques en 3 ' du gène & $\begin{array}{l}\text { Régulation de l'exocytose des neuro- } \\
\text { médiateurs }\end{array}$ & $\begin{array}{l}\text { Oui (1 SNP), OR = 1,15 [42] } \\
\text { Oui, OR = 1,19 [46] }\end{array}$ \\
\hline CHRNA4 & $\begin{array}{l}\text { Polymorphisme de répétitions de dinucléotides dans } \\
\text { l'intron } 1.7 \text { SNP dans les exons } 2 \text { et } 5\end{array}$ & $\begin{array}{l}\text { Sous-unité alpha } 4 \text { du récepteur de } \\
\text { l'acétylcholine }\end{array}$ & Non \\
\hline GRIN2A & SNP dans l'exon 5 & $\begin{array}{l}\text { Récepteur ionotropique au glutamate } \\
\text { dont le gène code une sous-unité du } \\
\text { récepteur NMDA }\end{array}$ & Non \\
\hline BDNF & $\begin{array}{l}\text { Substitution d'un acide aminé (Val en Met) au } \\
\text { codon } 66 \text {, allèle Val66 }\end{array}$ & $\begin{array}{l}\text { Protéine stimulant la croissance et la } \\
\text { différenciation des neurones }\end{array}$ & Non \\
\hline
\end{tabular}

Tableau III. Liste des gènes associés sur la base d'une étude d'association positive, répliquée au moins une fois en précisant si cette association a été confirmée par une méta-analyse (en choisissant les plus récentes). La plupart des gènes étudiés sont impliqués dans les systèmes de neurotransmission catécholaminergique et sérotoninergique. Un certain nombre de ces gènes ont fait l'objet de modèles animaux (voir Tableau II). DBH : dopamine-bétahydroxylase; MAO-A : monoamine oxidase A; COMT : catéchol-0-méthyltransférase; ADRA2A : récepteur adrénergique alpha-2-A; $D D R A 2 C$ : récepteur adrénergique alpha-2-C; TPH-2 : tryptophane hydroxylase; SNAP 25 : protéine synaptosomale de 25kD; CHRNA4 : récepteur nicotinique alpha 4 ; GRIN2A : glutamate receptor, ionotropic, $N$-méthyl D-aspartate $2 A$; NMDA : N-méthyl D-aspartate; BDNF brain-derived neurotrophic factor. 
(CHRNA4, cholinergic receptor nicotinic alpha 4, DATI et DRD4) comme le propose le modèle synaptique de Todd et Neuman [30]. La nicotine favoriserait la libération de DA dans le cortex préfrontal. Or, lors du développement cortical, la dopamine possède des effets morphogénétiques médiés par le récepteur dopaminergique D4, induisant des modifications de la maturation neuronale chez le fœtus.

\section{Études d'imagerie}

\section{Imagerie structurale}

Des études morphométriques réalisées chez les enfants atteints de TDAH montrent des atteintes plus généralisées que ce qui était attendu selon les données physiopathologiques impliquant les circuits fronto-striataux [31]. Des diminutions de volume du cerveau (les différents lobes; le noyau caudé) ainsi que du cervelet ont été mises en évidence dans de nombreuses études. Une méta-analyse basée sur 22 études individuelles a confirmé cette donnée [32]. Des études récentes sur une cohorte importante (223 enfants présentant un TDAH et 223 enfants contrôles) ont mis en évidence un retard de maturation corticale chez les patients TDAH, particulièrement prononcé au niveau du cortex préfrontal [33]. Ce retard de maturation est estimé à 3 ans : l'épaisseur corticale chez les patients TDAH de 10,5 ans est similaire à celle observée chez les enfants contrôles âgés de 7,5 ans. Cette étude montre l'exis- tence d'anomalies développementales d'un type particulier, puisque s'il n'y a pas de déviance démontrée dans la maturation corticale, il y a cependant un délai de maturation très significatif (le temps moyen nécessaire pour atteindre $50 \%$ du maximum d'épaisseur est de 7,5 ans chez les témoins contre 10,6 ans chez les enfants atteints de TDAH, $\left.p<1,0 \times 10^{-20}\right)$.

\section{Imagerie fonctionnelle}

Les études d'imagerie fonctionnelle ont principalement étudié des tâches cognitives liées au contrôle de l'attention, à la mémoire de travail et à l'inhibition de réponse. Une méta-analyse récente de ces travaux montre un défaut d'activation significatif du cortex préfrontal gauche, du cortex cingulaire antérieur, du lobe pariétal droit, du cortex occipital et du thalamus chez des sujets TDAH comparés aux contrôles [34]. Généralement, les sujets ayant un TDAH montrent des dysfonctionnements frontaux au cours des tâches impliquant l'inhibition de réponse et temporo-pariétaux pour celles impliquant l'attention. Par ailleurs, les sujets TDAH ont généralement une activation réduite du striatum en IRM (imagerie en résonance magnétique) fonctionnelle et montrent également une moindre activation d'un réseau striatocingulaire ventral au cours de tâches de motivation mettant en jeu un renforcement [35]. L'étude en imagerie des effets du traitement montre dans certains cas une normalisation de l'excès de densité striatale du DAT, une caractéristique mise en évidence chez des sujets TDAH avant l'exposition aux psychostimulants, mais dont les tentatives de réplication ont donné des résultats variables. L'imagerie fonctionnelle couplée à l'étude des génotypes montre que certains allèles de vulnérabilité du TDAH seraient impliqués dans les performances aux tests de mémoire de travail et dans les patrons d'activation chez les sujets
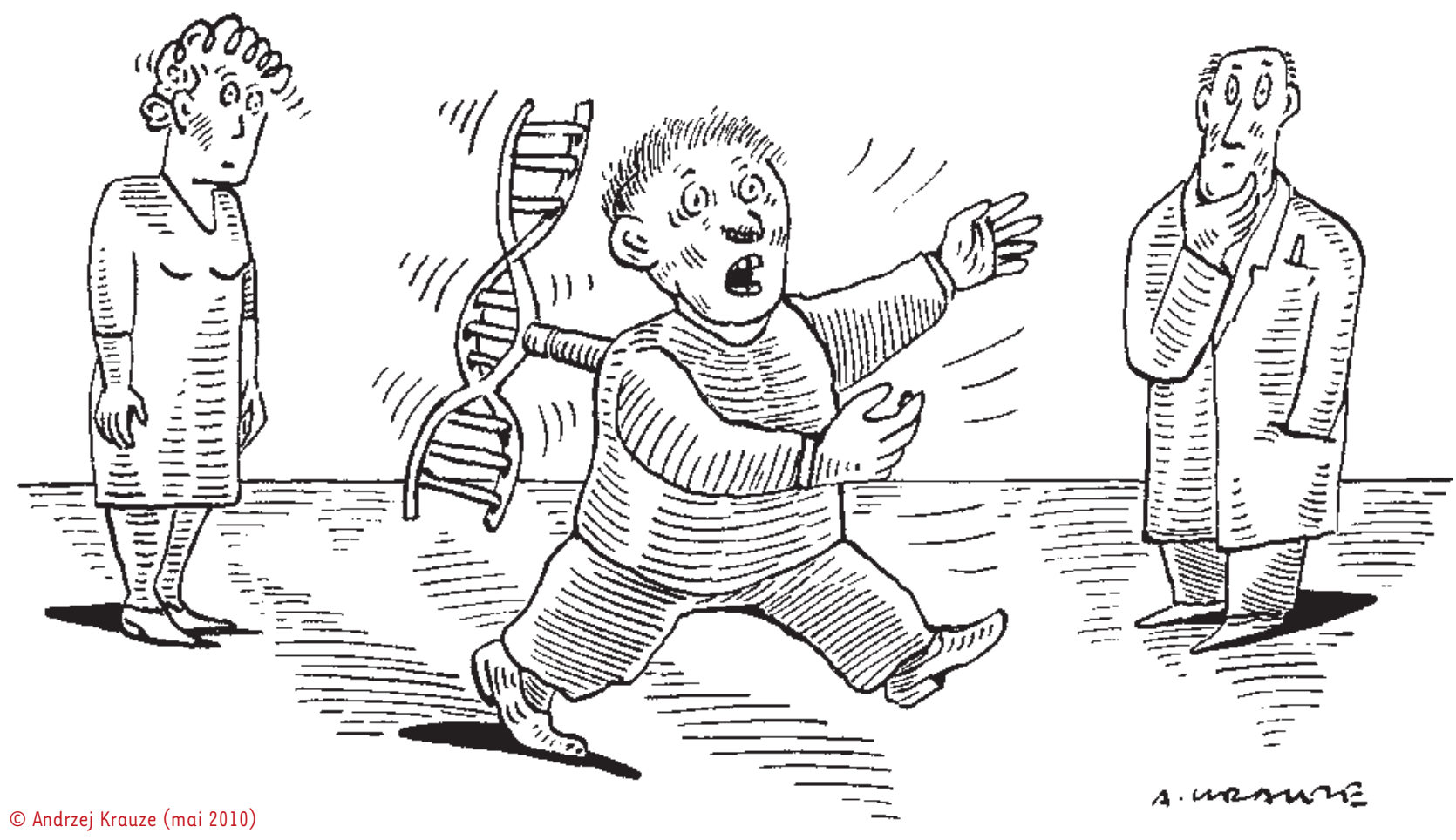

A. unanat

(C) Andrzej Krauze (mai 2010) 
sains. Ainsi, une baisse d'activité dans le cortex cingulaire antérieur a été trouvée en IRM fonctionnelle chez les sujets sains, en fonction du nombre d'allèles Met du gène codant pour l'enzyme responsable de la dégradation des catécholamines (polymorphisme Val158Met du gène COMT). Des résultats similaires ont été observés pour l'allèle $10-R$ de DATI. Les données issues de l'imagerie structurale et fonctionnelle ouvrent des pistes intéressantes pour l'exploration de nouveaux mécanismes étiopathogéniques du TDAH (par exemple le rôle possible des neurotrophines dans le délai de maturation corticale) mais ne permettent pas seules de conclure à une implication causale. En effet, les anomalies observées peuvent être primaires ou secondaires à des processus adaptatifs.

\section{Conclusion}

Les approches neurobiologiques du TDAH vont dans le sens d'une hétérogénéité étiologique du trouble. Si les facteurs génétiques y tiennent une place importante, les données actuelles sont en faveur de l'existence de locus multiples, chacun associé à un risque faible, mais aussi d'effets génétiques additifs et d'interactions gène-gène et gène-environnement. Il est probable que, dans un futur proche, les réseaux collaboratifs et le perfectionnement des techniques permettent de corréler des données morphologiques et fonctionnelles avec les polymorphismes des gènes de vulnérabilité du TDAH et de recueillir des données intégrant les différents niveaux d'expression clinique ainsi que la dimension développementale du trouble. $\diamond$

\section{SUMMARY}

\section{Neurobiology of attention}

\section{deficit/hyperactivity disorder}

Attention deficit/hyperactivity disorder (ADHD) is a frequent and disabling condition in school children, with cognitive and behavioral symptoms persisting into adulthood in a majority of patients. Etiology of ADHD is considered multifactorial and heterogenous, with an important contribution of genetic factors. Apart from genetic risk factors, emphasis has been put on the early environment, and prenatal exposure to nicotine, alcohol, prematurity and low birth weight have been associated with subsequent ADHD symptoms. This article reviews recent findings in neurobiology, genetics and neuroimaging of ADHD. Despite their clinical heterogeneity and frequent comorbidities, key symptoms of ADHD, such as impulsivity, hyperactivity and inattention are regularly improved by dopaminergic agonists, leading to consider dopaminergic dysfunction a possibly contributing factor in ADHD. Norepinephrine agonists also have clinical efficacy on ADHD symptoms and several other neurotransmission systems are likely involved in the etiology of ADHD. Dysfunction of neurotransmitter systems have been related to impairments of sustained attention, inhibitory control and working memory. Cognitive tasks focusing on reaction time and verbal working memory fit certain criteria for ADHD endophenotypes, offering a pathway to bridge the gap between observed traits and genetic vulnerability. Despite ADHD being a highly heritable disorder, most candidate genes with replicated findings across association studies only account for a small proportion of genetic variance. Neuroimaging studies using treatment effect or cognitive tasks show differential activation patterns in ADHD patients, with trends towards normalization under treatment. Further insight into neurobiological mechanisms involved in ADHD will arise from collaborative networks and combination of imaging, genetic and neurobiological techniques with consideration of the developmental aspects of ADHD. $\diamond$

\section{CONFLIT D'INTÉRÊTS}

Les auteurs déclarent n'avoir aucun conflit d'intérêts concernant les données publiées dans cet article.

\section{RÉFÉRENCES}

1. Polanczyk G, de Lima MS, Horta BL, et al. The worldwide prevalence of ADHD: a systematic review and metaregression analysis. Am J Psychiatry 2007; $164: 942-8$.

2. Spencer TJ, Biederman J, Mick $\varepsilon$. Attention-deficit/hyperactivity disorder: diagnosis, lifespan, comorbidities, and neurobiology. Ambul Pediatr 2007 ; 7 (suppl 1) : 73-81.

3. Mannuzza S, Klein RG, Bessler A, et al. Adult psychiatric status of hyperactive boys grown up. Am J Psychiatry 1998; 155 : 493-8.

4. Galéra C, Melchior M, Chastang JF, et al. Childhood and adolescent hyperactivity-inattention symptoms and academic achievement 8 years later: the GAZEL Youth study. Psychol Med 2009 ; 39 : 1895-906.

5. Mannuzza S, Klein RG, Moulton JL $3^{\text {rd }}$. Lifetime criminality among boys with attention deficit hyperactivity disorder: a prospective follow-up study into adulthood using official arrest records. Psychiatr Res 2008; 160 : 237-46.

6. Faraone SV, Doyle AE. The nature and heritability of attention-deficit/ hyperactivity disorder. Child Adolesc Psychiatr Clin North Am 2001; 10 : 299-316, viii-ix.

7. Langley K, Rice F, van den Bree MB, Thapar A. Maternal smoking during pregnancy as an environmental risk factor for attention deficit hyperactivity disorder behaviour. Minerva Pediatr 2005 ; 57 : 359-71.

8. Thapar A, Fowler T, Rice F, et al. Maternal smoking during pregnancy and attention deficit hyperactivity disorder symptoms in offspring. Am J Psychiatry 2003; $160: 1985-9$.

9. Mill J, Petronis A. Pre- and peri-natal environmental risks for attentiondeficit hyperactivity disorder (ADHD): the potential role of epigenetic processes in mediating susceptibility. J Child Psychol Psychiatry 2008 ; 49: 1020-30.

10. Sonuga-Barke $\varepsilon$ J. Causal models of attention-deficit/hyperactivity disorder: from common simple deficits to multiple developmental pathways. Biol Psychiatry $2005 ; 57$ : 1231-8.

11. Rommelse NN, Arias-Vásquez A, Altink ME, et al. Neuropsychological endophenotype approach to genome-wide linkage analysis identifies susceptibility loci for ADHD on 2q21.1 and 13q12.11. Am J Hum Genet 2008: 83: 99-105.

12. Volkow ND, Wang GJ, Fowler JS, Ding YS. Imaging the effects of methylphenidate on brain dopamine: new model on its therapeutic actions for attention-deficit/hyperactivity disorder. Biol Psychiatry 2005; $57: 1410-5$.

13. Sagvolden T, Johansen EB, Aase H, Russell VA. A dynamic developmental theory of attention-deficit/hyperactivity disorder (ADHD) predominantly hyperactive/impulsive and combined subtypes. Behav Brain Sci 2005 ; 28 : 397-419; discussion 419-68.

14. Easton N, Shah YB, Marshall FH, et al. Guanfacine produces differential effects in frontal cortex compared with striatum: assessed by phMRI BOLD contrast. Psychopharmacology (Berl) 2006 ; 189: 369-85.

15. Brennan AR, Arnsten AF. Neuronal mechanisms underlying attention deficit hyperactivity disorder: the influence of arousal on prefrontal cortical function. Ann NY Acad Sci 2008 ; 1129 : 236-45.

16. Gonon F. The dopaminergic hypothesis of attention-deficit/hyperactivity disorder needs re-examining. Trends Neurosci $2009 ; 32: 2-8$. 
17. Van der Kooij MA, Glennon JC. Animal models concerning the role of dopamine in attention-deficit hyperactivity disorder. Neurosci Biobehav Rev 2007 ; 31 : 597-618.

18. DasBanerjee T, Middleton FA, Berger DF, et al. A comparison of molecular alterations in environmental and genetic rat models of ADHD: a pilot study. Am J Med Genet B Neuropsychiatr Genet 2008 ; 147B : 1554-63.

19. Zhou K, Chen W, Buitelaar J, et al. Genetic heterogeneity in ADHD: DATl gene only affects probands without CD. Am J Med Genet 2008; 147B : 1481-7.

20. Neale $B$, Faraone $S$. Perspective on the genetics of attention deficit/ hyperactivity disorder. Am J Med Genet 2008; 147B : 1334-6.

21. Zhou K, Dempfle A, Arcos-Burgos M, et al. Meta-analysis of genome-wide linkage scans of attention deficit hyperactivity disorder. Am J Med Genet 2008; 147B : 1392-8

22. Manolio TA, Collins FS, Cox NJ, et al. Finding the missing heritability of complex diseases. Nature $2009 ; 461: 747-53$.

23. Tsankova N, Renthal W, Kumar A, Nestler $\varepsilon$ J. Epigenetic regulation in psychiatric disorders. Nat Rev Neurosci $2007 ; 8: 355-67$.

24. Mill J, Richards S, Knight J, et al. Haplotype analysis of SNAP-25 suggests a role in the aetiology of ADHD. Mol Psychiatry $2004 ; 9: 801-10$.

25. Winsberg $B G$, Comings $D E$. Association of the dopamine transporter gene (DATI) with poor methylphenidate response. J Am Acad Child Adolesc Psychiatry $1999 ; 38$ : 1474-7.

26. Purper-Ouakil D, Wohl M, Orejarena $S$, et al. Pharmacogenetics of methylphenidate response in attention deficit/hyperactivity disorder: Association with the dopamine transporter gene (SLC6A3). Am J Med Genet Neuropsychiatr Genet 2008; 147B : 1425-30.

27. McGough J, McCracken J, Swanson J, et al. Pharmacogenetics of methylphenidate response in preschoolers with ADHD. J Am Acad Child Adolesc Psychiatry 2006 ; 45 : 1314-22.

28. Seeger G, Schloss P, Schmidt MH. Functional polymorphism within the promotor of the serotonin transporter gene is associated with severe hyperkinetic disorders. Mol Psychiatry $2001 ; 6$ : 235-8.

29. Neuman RJ, Lobos $\varepsilon$, Reich W, et al. Prenatal smoking exposure and dopaminergic genotypes interact to cause a severe ADHD subtype. Biol Psychiatry $2007 ; 61: 1320-8$.

30. Todd RD, Neuman RJ. Gene-environment interactions in the development of combined type ADHD: evidence for a synapse-based model. Am J Med Genet Neuropsychiatr Genet 2007; 144B : 971-5.

31. Bush G. Attention-deficit/hyperactivity disorder and attention networks. Neuropsychopharmacology $2010 ; 35: 278-300$
32. Valera $\varepsilon M$, Faraone SV, Murray KE, Seidman LJ. Meta-analysis of structural imaging findings in attention-deficit/hyperactivity disorder. Biol Psychiatry 2007 ; 61 : 1361-9.

33. Shaw P, Eckstrand K, Sharp W, et al. Attention-deficit/hyperactivity disorder is characterized by a delay in cortical maturation. Proc Natl Acad Sci USA 2007 ; 104 : 19649-54.

34. Dickstein SG, Bannon K, Castellanos FX, Milham MP. The neural correlates of attention deficit hyperactivity disorder: an ALE meta-analysis. J Child Psychol Psychiatry 2006 ; 47 : 1051-62.

35. Paloyelis Y, Mehta MA, Kuntsi J, Asherson P. Functional MRI in ADHD: a systematic literature review. Exp Rev Neurother 2007 ; 7 : 1337-56.

36. Sagvolden T. Behavioral validation of the spontaneously hypertensive rat (SHR) as an animal model of attention-deficit/hyperactivity disorder (AD/HD). Neurosci Biobehav Rev 2000 ; $24: 31-9$.

37. Heyser CJ, Wilson MC, Gold LH. Coloboma hyperactive mutant exhibits delayed neurobehavioral developmental milestones. Brain Res Dev Brain Res 1995; 89 : 264-9.

38. Gainetdinov RR, Caron MG. An animal model of attention deficit hyperactivity disorder. Mol Med Today $2000 ; 6: 43-4$

39. Besson M, Suarez S, Cormier A, et al. Chronic nicotine exposure has dissociable behavioural effects on control and beta2 ${ }^{-/-}$mice. Behav Genet 2008 ; 38 : 503-14.

40. Moon J, Beaudin AE, Verosky $S$, et al. Attentional dysfunction, impulsivity, and resistance to change in a mouse model of fragile X syndrome. Behav Neurosci $2006 ; 120$ : 1367-79.

41. Li D, Sham PC, Owen MJ, He L. Meta-analysis shows significant association between dopamine system genes and attention deficit hyperactivity disorder (ADHD). Hum Mol Genet 2006; $15: 2276-84$

42. Gizer IR, Ficks C, Waldman ID. Candidate gene studies of ADHD: a meta-analytic review. Hum Genet $2009 ; 126: 51-90$.

43. Yang B, Chan RC, Jing J, et al. A meta-analysis of association studies between the 10 -repeat allele of a VNTR polymorphism in the 3'-UTR of dopamine transporter gene and attention deficit hyperactivity disorder. Am J Med Genet Neuropsychiatr Genet 2007 ; 144B : 541-50.

44. Smoller JW, Biederman J, Arbeitman L, et al. Association between the $5 \mathrm{HTlB}$ receptor gene (HTRIB) and the inattentive subtype of ADHD. Biol Psychiatry $2006 ; 59: 460-7$.

45. Faraone SV, Perlis RH, Doyle AE, et al. Molecular genetics of attention-deficit/hyperactivity disorder. Biol Psychiatry 2005; $57: 1313-23$.

46. Mick $\varepsilon$, Faraone SV. Genetics of attention deficit hyperactivity disorder. Child Adolesc Psychiatr Clin North Am $2008 ; 17: 261-84$

\section{Bon de commande}

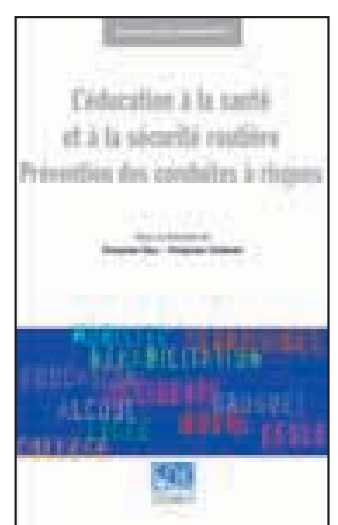

ISBN : 978-2-8425-4138-5 438 pages

À retourner à EDK, 2, rue Troyon - 92316 Sèvres Cedex

Tél. : 0155641393 - Fax : 0155641394 - E-mail : edk@edk.fr

NOM :

Prénom :

Adresse :

Code postal :

Ville :

Pays :

Fonction :

Je souhaite recevoir l'ouvrage $\mathbf{L}$ 'éducation à la santé et à la sécurité routière $: 20 €+3 €$ de port $=\mathbf{2 3} € \mathbf{T T C}$ en ................ exemplaire, soit un total de $€$

$\square$ Par chèque, à l'ordre de $\mathbf{E} \mathbf{D} \mathbf{K}$

$\square$ Par carte bancaire : $\square$ Visa $\square$ Eurocard/Mastercard

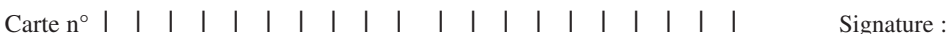

Date d'expiration: $\quad|\quad| 1|1|$

$\mathrm{N}^{\circ}$ de contrôle au dos de la carte : $\quad|\quad| \quad \mid$

TIRÉS À PART

M. Simonneau 